Ciência Florestal, Santa Maria, v. 23, n. 3, p. 305-315, jul.-set., 2013

ISSN 0103-9954

\title{
FATORES DO MEIO FÍSICO ASSOCIADOS AO ESTABELECIMENTO DE ESPÉCIES RÚSTICAS EM ECOSSISTEMAS PERTURBADOS NA MATA ATLÂNTICA, PIRAÍ, RJ - BRASIL $^{1}$
}

\author{
ECOLOGICAL FACTORS ASSOCIATED WITH THE ESTABLISHMENT OF RUSTIC SPECIES \\ IN DISTURBED ECOSYSTEMS IN THE ATLANTIC FOREST, PIRAÍ, RIO DE JANEIRO STATE - \\ BRAZIL
}

\author{
Hiram Feijó Baylão Junior ${ }^{2}$ Ricardo Valcarcel ${ }^{3}$ Felipe Cito Nettesheim ${ }^{4}$
}

\begin{abstract}
RESUMO
Ecossistemas perturbados não apresentam composição florística original, os solos encontram-se exauridos, rasos, pedregosos, com baixa infiltração, revelando processos erosivos com diferentes níveis de geodinamismo. A área de estudo apresenta pastagens com vegetação herbácea espaçada e fragilizada a cada chuva ou incêndio, onde os indivíduos florestais que colonizaram e se estabeleceram nesses ambientes foram definidos como espécies rústicas. Este estudo objetivou relacionar as variáveis do meio físico (exposição, declividade, elevação, relevo e afloramentos rochosos) com o estabelecimento e crescimento das espécies rústicas levantadas e identificadas por censo, em trecho perturbado (terço inferior) da bacia do rio Cacaria, situado na base da serra do Mar, Piraí - RJ. Para o levantamento da vegetação foi realizado censo em uma área de 22 hectares, onde foram mensuradas, identificadas e georreferenciadas todas as espécies arbóreas de ocorrência espontânea que se encontravam isoladas em uma área de pastagem. Os fatores ecológicos exposição, elevação e declividade foram determinados com bússola, altímetro e clinômetro, respectivamente. Foram levantados e identificados 131 indivíduos, constituindo 14 espécies, agrupadas em 9 famílias. Tabernaemontana laeta Mart., Sparattosperma leucanthum (Vell.) Schum., Machaerium hirtum (Vell.) Stellfeld, Tabebuia chrysotricha (Mart. ex DC.) Stan., Cecropia pachystachya Trec., Peltophorum dubium (Spreng.) Taub., Guarea guidonia (L.) Sleumer, Acacia polyphylla DC. e Psidium guajava L. estiveram presentes em trechos da encosta com exposição para o norte, com cotas altitudinais de $60 \mathrm{~m}$ a $80 \mathrm{~m}$ e com declividade fortemente ondulada (20-45\%), indicando preferência dessas espécies por microhabitats com essas características.
\end{abstract}

Palavras-chave: florística; ecossistemas perturbados; restauração.

\begin{abstract}
Disturbed ecosystems do not present original floristic composition. Their soils are depleted, shallow, stony, with low infiltration and present erosion with different levels of geodynamics. The study region has areas of pasture with sparse herbaceous vegetation which is weakened in every rain and fire, covering less ground. The individuals that colonized and settled in these environments were considered rustic species. This study raised and identified by census, the rustic species and their structure in the most degraded part (lower third) of the watershed of Cacaria's river at the base of the Serra do Mar, Piraí, Rio de Janeiro state, and evaluated the influence of ecological exposure, slope, elevation, topography and rock outcrops in the establishment and growth of these species. For the vegetation survey it was conducted census in an area of 22 hectares, where it was measured, geo-referenced and identified all spontaneous tree species that were isolated in a

1 Parte da Dissertação de mestrado do primeiro autor apresentada ao Programa de Pós-Graduação em Ciências Ambientais e Florestais da Universidade Federal Rural do Rio de Janeiro.

2 Engenheiro Florestal, MSc., Laboratório de Manejo de Bacias Hidrográficas, Departamento de Ciências Ambientais, Instituto de Florestas, Universidade Federal Rural do Rio de Janeiro, Caixa Postal 74529, CEP 23851-970, Seropédica (RJ). baylaojr@globomail.com

3 Engenheiro Florestal, Dr., Professor Associado III do Departamento de Ciências Ambientais, Instituto de Florestas, Universidade Federal Rural do Rio de Janeiro, CEP 23851-970, Seropédica (RJ). ricval@ufrrj.br

4 Biólogo, MSc., Programa de Pós-Graduação em Ecologia, Universidade Federal do Rio de Janeiro, CEP 23851-970, Seropédica (RJ).

Recebido para publicação em 21/06/2011 e aceito em 16/11/2011
\end{abstract}


pasture area. Ecological factors exposure, elevation and slope were determined with a compass, altimeter and clinometer, respectively. There were identified 131 individuals, representing 14 species, grouped into nine families. Tabernaemontana laeta Mart., Sparattosperma leucanthum (Vell.) Schum., Machaerium hirtum (Vell.) Stellfeld, Tabebuia chrysotricha (Mart. ex DC.) Stan., Cecropia pachystachya Trec., Peltophorum dubium (Spreng.) Taub., Guarea guidonia (L.) Sleumer, Acacia polyphylla DC. and Psidium guajava L.were present in portions of the slope with exposure to the north, with altitudes from 60 to $80 \mathrm{~m}$ and with slope strongly corrugated (20-45\%), indicating a preference of these species for microhabitats with those characteristics.

Keywords: floristic; perturbed ecosystems; restoration.

\section{INTRODUÇÃO}

Espécies rústicas são aquelas capazes de colonizar barrancos, terrenos que perderam capacidade de produção agrícola, pastagens ainda em uso, estabelecendo-se e se desenvolvendo em terrenos declivosos, com solos rasos, baixa infiltração, pedregosos e quimicamente pobres em nutrientes (POTT e POTT, 2002). Estas habilidades constituem um fator diferencial para que essas espécies possam ser utilizadas como medidas biológicas de restauração florestal, capazes de reverter tendência inercial de degradação (CORTINES e VALCARCEL, 2009), passando para tendência de restauração, se as mesmas conseguirem desenvolver mecanismos de facilitação capazes de propiciar a entrada de novas espécies.

Durante a sucessão, a distribuição natural das espécies sofre influência de fatores ambientais, onde barreiras físicas podem operar como filtros catalisadores e/ou reguladores para a dispersão e estabelecimento das mesmas espécies (ESPINOLA e JULIO JUNIOR, 2007). Tais barreiras variam na paisagem, gerando alterações de habitat que parecem influenciar as formações vegetais. De fato, alternâncias na topografia vêm sendo consideradas importantes fontes de variações na distribuição espacial de espécies vegetais (SVENNING et al., 2004; JONES et al., 2008). Aparentemente, estas diferenças causam variações de fatores ecológicos determinantes para o estabelecimento das plantas (como mudanças nas propriedades dos solos, regime hídrico e fertilidade), contribuindo para definir a estrutura e composição de florestas tropicais (RODRIGUES et al., 2007). A heterogeneidade ambiental de ecossistemas florestais nos trópicos é, portanto, o resultado da integração dessa diversidade de fatores ecológicos que influenciam e são influenciados pelo contexto espaço-temporal no qual estão inseridos (BISPO et al., 2009).

As espécies e o seu desenvolvimento sin- tetizam as complexas inter-relações ambientais. Conhecer os seus padrões de distribuição e a estruturação dos mosaicos florestais é um passo importante para a restauração de ecossistemas perturbados, tornando-se relevante em áreas com características ambientais singulares, como matas de galeria, regiões com solos de alta erodibilidade, compactados pela pecuária ou exauridos e abandonados pela agricultura (GIEHL et al., 2007). Sendo este um cenário recorrente na degradada Mata Atlântica (DEAN, 1996), o conhecimento da biologia de espécies que colonizam vertentes, com baixa oferta de atributos ambientais, pode facilitar a regeneração e sucessão natural, tendo potencial de constituir estratégias de restauração e recuperação de ecossistemas perturbados (LONGHI et al, 2009).

Assumindo que o conhecimento sobre os processos ecológicos e biológicos das espécies envolvidas na dinâmica de comunidades vegetais são condicionantes básicos para a conservação, restauração e manejo de ecossistemas naturais, o presente estudo se propõe a: (1) caracterizar a estrutura de espécies arbóreas rústicas de potencial facilitador em um ecossistema perturbado de Mata Atlântica; (2) investigar a existência de padrões ecológicos ligados à variação ambiental que possam determinar a ocorrência de espécies rústicas em ecossistemas perturbados.

\section{MATERIAL E MÉTODOS}

\section{Área de Estudo}

A área de estudo está localizada na bacia hidrográfica do rio Cacaria, distrito de Cacaria, município de Piraí, estado do Rio de Janeiro. O levantamento dos dados foi realizado no Sítio Três Irmãs em uma encosta de 22 hectares (coordenadas UTM: E - 621.045,499 e N - 7.488.921,327) com orientação geográfica predominantemente norte, a qual está situada entre várzeas com pecuária exten- 
siva e fragmentos florestais nas partes altas da base da Serra das Araras, denominação local da Serra do Mar (Figura 1). Embora a exposição norte seja predominante, há pequenos trechos com exposição noroeste e nordeste. No local, o relevo ondulado apresenta declividades superiores a $45^{\circ}$, entremeados por áreas menos declivosas. A área de estudo já sofreu diversas intervenções antrópicas e apresenta características de uso intenso da encosta por pecuária extensiva em decrepitude e incidência de queimadas anuais, havendo vários indícios de esgotamento dos seus solos. Esta área foi selecionada como modelo, pois caracteriza fisionomicamente a situação típica da paisagem regional.

\section{Amostragem}

\section{Levantamento estrutural de espécies rústicas}

A densidade das espécies arbóreas de ocorrência espontânea na área de estudo é baixa. Portanto, optou-se por realizar um censo, incluindo

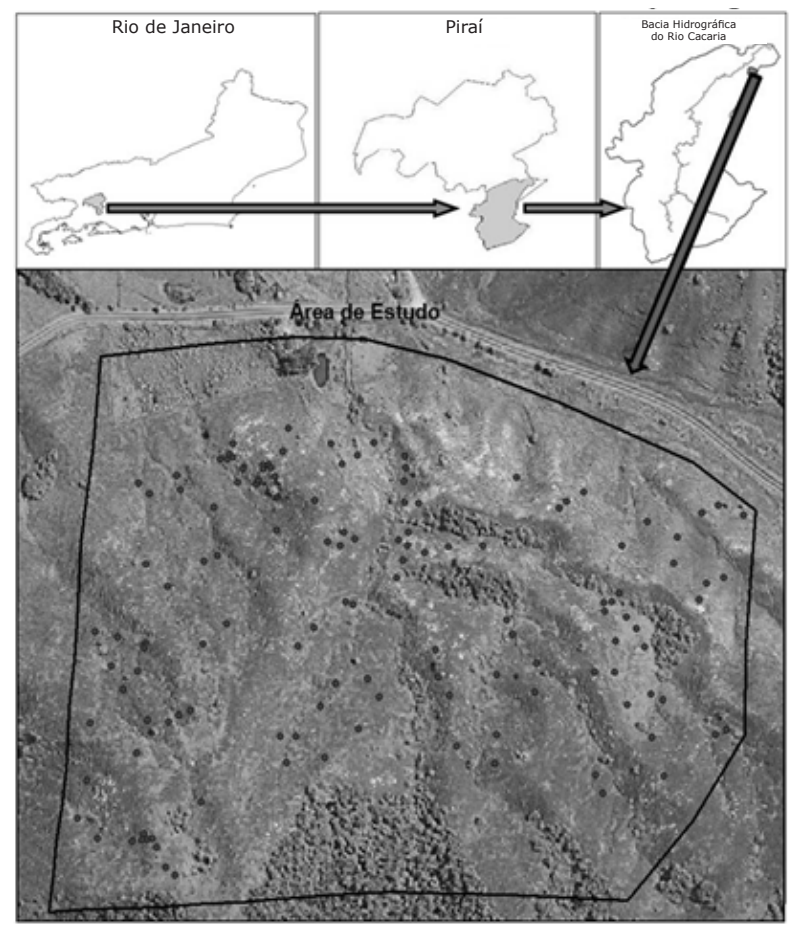

FIGURA 1: Área de estudo com a distribuição dos indivíduos (pontos) que conseguiram colonizar e se estabelecer no terço inferior da bacia do Rio Cacaria.

FIGURE 1: Study area with the distribution of individuals who have managed to colonize and settle in the lower third of the Cacaria river watershed. no levantamento todos os indivíduos com CAP > $15 \mathrm{~cm}$. A amostragem foi limitada entre o fim da várzea e o inicio do topo dos morros, entre as cotas de elevação $60 \mathrm{~m}-200 \mathrm{~m}$ acima do nível do mar. Isso foi feito para minimizar efeitos do pastoreio (concentrado nas partes centrais da várzea) e dos fragmentos florestais situados nos locais mais elevados da área. Todos os indivíduos amostrados tiveram seu CAP medido com fita métrica e foram georreferenciados (GPS Garmin modelo 76csx), coletados, etiquetados e armazenados em sacos plásticos. $\mathrm{O}$ material foi levado para o Laboratório de Manejo de Bacias Hidrográficas (LMBH) da Universidade Federal do Rural do Rio de Janeiro (UFRRJ), sendo herborizado (MORI et al., 1985) e identificado por meio de comparação com exsicatas do Herbário da UFRRJ (RBR), ou consulta a especialistas. O sistema de classificação adotado foi sugerido no APG II (2003). Partindo dos dados quantitativos coletados no censo, a descrição estrutural das espécies rústicas foi feita através do cálculo dos parâmetros fitossociológicos (MATTEUCCI e COLMA, 1982) de Densidade Relativa (DR), Dominância Relativa (DoR) e Valor de Cobertura (VC) foram realizados no software Microsoft Office Excel 2007.

\section{Obtenção de dados sobre fatores ecológicos}

Considerando que cada espécie era um tratamento e os indivíduos, repetições de unidades amostrais (UAs), estas foram georreferenciadas, sendo identificadas e localizadas sobre um modelo digital do terreno, originado de bases 1:50.000 do IBGE e usos oriundos da restituição da imagem IKONOS do ano de 2002. Também foi gerado um Modelo Digital de Elevação (MDE), que permitiu criar mapas que forneceram dados abióticos. Isso foi feito através do software ArcGis 9.3 e de sua ferramenta "surface" (SCHMIDT et al., 2003). Desta forma, e com alguns dados coletados no local, foram registrados dados abióticos potencialmente importantes para determinar a ocorrência e estabelecimento de espécies rústicas em ecossistemas degradados. Assim, em um raio de $5 \mathrm{~m}$ de cada UA, foram coletadas informações sobre o microrrelevo, afloramentos rochosos, elevação e declividade. O microrrelevo no entorno de cada indivíduo foi diferenciado visualmente entre calha (favorece o escoamento natural da água aumentando a frequência de umidade nessas áreas, porém, são vulneráveis aos processos erosivos e movimentos de massa), colúvio (favorece o acúmulo de material orgânico, 
constituindo manto de substrato profundo e com capacidade de armazenar umidade) ou meia-encosta (apresenta vocação dispersora de água e baixa capacidade de armazenamento de umidade e nutrientes). A existência de afloramentos rochosos foi considerada como a exposição natural em superfície, de rocha ou mineral, acessíveis à observação humana (IBGE, 2004), sendo registrada sua presença ou ausência. A elevação foi obtida primeiramente através do MDE da área, sendo o dado refinado pelo uso no local de um altímetro de precisão e posteriormente reclassificado em intervalos de classe (Tabela 1). A declividade foi obtida em \% de inclinação através do MDE, sendo refinada com auxílio de um clinômetro, e em seguida reclassificada em intervalos de classe (Tabela 2) segundo o Sistema Brasileiro de Classificação dos Solos (EMBRAPA, 1997).

TABELA 1: Classificação de elevação do terreno aplicada às áreas perturbadas do terço inferior da bacia hidrográfica do rio Cacaria, Piraí - RJ.

TABLE 1: Classification of terrain elevation Applied to disturbed areas of the lower third of the watershed of Cacaria's river, Piraí, RJ state.

\begin{tabular}{cc}
\hline Classes de elevação & Elevação $(\mathrm{m})$ \\
\hline 1 & $<60$ \\
2 & $60-80$ \\
3 & $80-100$ \\
4 & $100-120$ \\
5 & $120-140$ \\
6 & $140-160$ \\
7 & $160-180$ \\
8 & $180-200$ \\
\hline
\end{tabular}

TABELA 2: Classificação de declividade (EMBRAPA, 1997).

TABLE 2: Slope classification (EMBRAPA, 1997).

\begin{tabular}{cc}
\hline Classes de declividade & $\%$ \\
\hline Plano & $0-3$ \\
Suavemente ondulado & $3-8$ \\
Ondulado & $8-20$ \\
Fortemente ondulado & $20-45$ \\
Montanhoso & $45-75$ \\
Fortemente montanhoso & $>75$ \\
\hline
\end{tabular}

\section{Análise dos dados}

Foi montada uma matriz, onde os indivíduos ocupavam as colunas e os respectivos fatores ecológicos (na forma de dados categorizados) preenchiam as linhas. Foram consideradas na análise apenas indivíduos (UAs) de espécies (tratamentos) que somavam mais de cinco repetições na paisagem. Devido às características do ambiente de estudo, à biologia das espécies e à metodologia empregada, não foi possível padronizar o número de repetições por tratamentos segundo um máximo ideal, sendo padronizado apenas o limite mínimo para inclusão na análise. Assim, foram consideradas na análise descrita adiante: Tabernaemontana laeta Mart., Sparattosperma leucanthum (Vell.) Schum., Machaerium hirtum (Vell.) Stellfeld, Tabebuia chrysotricha (Mart. ex DC.) Stan., Cecropia pachystachya Trec., Peltophorum dubium (Spreng.) Taub., Guarea guidonia (L.) Sleumer, Acacia polyphylla DC. A matriz de dados foi utilizada para calcular a similaridade entre as repetições das unidades amostrais, utilizando o coeficiente de Bray-Curtis (MCCUNE e GRACE, 2002). Isso gerou uma nova matriz, contendo as similaridades entre as repetições das unidades amostrais (baseadas nos fatores ecológicos registrados em cada uma). Esta nova matriz de similaridade foi então usada como base para uma Análise Monotônica de Escalonamento Multidimensional (MMDS), realizada através do programa SYSTAT 11 (2005). Como os produtos da análise do MMDS, foram geradas: uma medida de ajuste (fitness) que é chamada Stress, outra que é a proporção de variância captada pela correlação ao quadrado (Squared correlation - RSQ ou R²) e a configuração bidimensional final da ordenação. Sobre a configuração bidimensional final, para sinalizar tendências comportamentais entre as diferentes espécies (tratamentos), foram desenhadas elipses bivariadas de Gauss. Para quantificar se houve diferenças na ordenação das espécies (tratamentos), expressas pelas elipses de Gauss, foi realizada uma análise de variância multivariada (MANOVA). A significância dos resultados da MANOVA foi testada por meio do teste de Pillai Trace.

Como análise exploratória adicional, buscando identificar os fatores ecológicos mais importantes na ordenação das UAs (indivíduos), foi utilizado o Coeficiente de Pearson. Assim, a configuração final das repetições das unidades amostrais (indivíduos) foi correlacionada com os dados de cada fator ecológico. Esta abordagem possibilita 
inferir sobre fatores ambientais com maior potencial para determinar os padrões de diferenciação encontrados na ordenação dos indivíduos de cada espécie. Apenas fatores ambientais que apresentaram correlação de Pearson $>0,5$ são apresentados e discutidos nas figuras e no texto.

\section{RESULTADOS E DISCUSSÃO}

Foram registrados 131 indivíduos, pertencentes a 14 espécies e agrupados em 9 famílias. As espécies de maior ocorrência foram Tabernaemontana laeta Mart. (30 indivíduos), Sparattosperma leucanthum (Vell.) Schum. (28), Machaerium hirtum (Vell.) Stellfeld. (17) e Tabebuia chrysotricha (Mart. ex DC.) Stan. (15). Juntas, apenas estas quatro espécies colonizadoras iniciais somam $68,7 \%$ dos indivíduos encontrados na área de estudo (Tabela 3). Isso demonstra a importância que essas espécies podem ter na restauração de ecossistemas perturbados na Mata Atlântica.

Das quatro espécies supracitadas, destacam-se Tabernaemontana laeta, Sparattosperma leucanthum e Machaerium hirtum, todas com valores de cobertura que somam quase o dobro ou mais (devido às elevadas densidade e dominância relativa) que as demais espécies registradas (Tabela 3). Tabernaemontana laeta é uma espécie que ocorre por todo sudeste brasileiro, geralmente associada a ambientes de elevada luminosidade e temperatura, como bordas de fragmentos e clareiras (MATOZINHOS e KONNO, 2008). Sparattosperma leucanthum é uma espécie amplamente distribuída no Brasil, ocorrendo em áreas secas na Amazônia, em vários tipos de formações vegetais na costa brasileira e apontada em estudo no Parque Nacional do Itatiaia como típica de ambientes com bastante exposição à luz, como bordas e encostas íngremes de floresta (GENTRY, 1992; PEREIRA e MANSANO, 2008). Por sua vez, Machaerium hirtum ocorre desde as savanas úmidas da Bolívia e Paraguai, até diferentes formações vegetais da Floresta Atlântica. Ela é uma planta decídua, pioneira e heliófita, comumente encontrada, assim como Tabernaemontana laeta e Sparattosperma leucanthum, em bordas de florestas e áreas de menor cobertura vegetal. Estas três espécies apresentam preferências ambientais bem semelhantes, além de possuírem dispersão anemocórica (Machaerium hirtum e Sparattosperma leucanthum) e zoocórica (Tabernaemontana laeta) (LORENZI, 2002). É possível que estas características facilitem sua dispersão e estabelecimento em áreas degradadas próximas a fragmentos florestais, onde espécies que dependem de outros tipos de dispersão e possuem outras preferências ambientais podem não conseguir chegar e se estabelecer.

TABELA 3: Espécies encontradas nas encostas de ecossistemas perturbados na bacia hidrográfica do rio Cacaria, Piraí - RJ. Legenda: ni - número de indivíduos; Dens. rel. - densidade relativa; Domi. rel. - dominância relativa; $\mathrm{VC}$ - valor de cobertura.

TABLE 3: Species found on the slopes in disturbed ecosystems of the watershed of Cacaria's river, Piraí, RJ state. Legend: ni - number of individuals; Dens. rel. - Relative density, Domi. rel. - Relative dominance; $\mathrm{VC}$ - value coverage.

\begin{tabular}{|c|c|c|c|c|c|}
\hline Espécie & Família & $\mathrm{ni}$ & Dens. rel. & Domi. rel. & $\mathrm{VC}$ \\
\hline Tabernaemontana laeta Mart. & Apocynaceae & 30 & 22,901 & 14,784 & 37,685 \\
\hline Sparattosperma leucanthum (Vell.) Schum. & Bignoniaceae & 28 & 21,374 & 16,410 & 37,784 \\
\hline Machaerium hirtum (Vell.) Stellfeld. & Fabaceae-Fab. & 17 & 12,977 & 18,480 & 31,458 \\
\hline Tabebuia chrysotricha (Mart. ex DC.) Stan. & Bignoniaceae & 15 & 11,450 & 6,440 & 17,891 \\
\hline Peltophorium dubium (Spreng.) Taub. & Fabaceae-Caes. & 9 & 6,870 & 11,185 & 18,056 \\
\hline Cecropia pachystachya Trec. & Urticaceae & 9 & 6,870 & 3,164 & 10,034 \\
\hline Guarea guidonia (L.) Sleumer & Meliaceae & 8 & 6,107 & 13,245 & 19,351 \\
\hline Acacia polyphylla DC. & Fabaceae-Mim. & 6 & 4,580 & 6,711 & 11,291 \\
\hline Psidium guajava $\mathrm{L}$. & Myrtaceae & 3 & 2,290 & 2,737 & 5,027 \\
\hline Coutarea hexandra (Jacq.) K. Schum. & Rubiaceae & 2 & 1,527 & 2,730 & 4,257 \\
\hline Sapium glandulatum (Vell.) Pax & Euphorbiaceae & 1 & 0,763 & 2,235 & 2,999 \\
\hline Cupania oblongifolia Mart. & Sapindaceae & 1 & 0,763 & 1,494 & 2,257 \\
\hline Apuleia leiocarpa (Vogel) J.F. Macbr. & Fabaceae-Caes. & 1 & 0,763 & 0,206 & 0,970 \\
\hline Myrocarpus fastigiatus Allemao & Fabaceae-Fab. & 1 & 0,763 & 0,178 & 0,941 \\
\hline Total & & 131 & 100,000 & 100,000 & 200,000 \\
\hline
\end{tabular}


Apesar de Tabebuia chrysotricha apresentar as mesmas características ecológicas que Tabernaemontana laeta, Sparattosperma leucanthum e Machaerium hirtum, inclusive com valores próximos de abundância e densidade relativa de Machaerium hirtum, ela possui baixa dominância relativa devido a sua pequena área basal, o que acarreta no baixo $\mathrm{VC}$ em relação às outras três espécies (Tabela 3). Considerando que esta espécie alcança até 10 metros de altura e $40 \mathrm{~cm}$ de diâmetro, é razoável supor que sua baixa dominância relativa possa indicar que esta espécie pode se estabelecer com facilidade, mas não se desenvolve em sua plenitude nas áreas degradadas. Situação parecida parece ocorrer com Peltophorum dubium e Guarea guidonia, onde estas duas espécies apresentam VC semelhante ao de Tabebuia chrysotricha, porém, com baixa densidade e alta dominância relativa (Tabela 3). Nesse caso, é possível que, ao contrário do que acontece com Tabebuia chrysotricha, estas duas espécies possuam maior dificuldade de se estabelecer, porém, uma vez estabelecidas, se desenvolvem bem e alcançam maiores áreas basais. No caso de Guarea guidonia é admissível que a suposta deficiência para se estabelecer em ambientes degradados seja consequência de sua síndrome de dispersão zoocórica, o que poderia dificultar a chegada de propágulos em áreas abertas. Ainda assim, é importante considerar também que o fato de Peltophorum dubium e Guarea guidonia alcançarem áreas basais maiores do que Tabebuia chrysotricha pode ser apenas consequência dessas duas espécies apresentarem, geralmente, maior porte. Independente dos menores VCs dessas três espécies em relação à Tabernaemontana laeta, Sparattosperma leucanthum e Machaerium hirtum, a aparente capacidade de se estabelecerem e se desenvolverem em áreas degradadas, ainda que não tão eficientemente, também as tornam boas opções em trabalhos de restauração de ecossistemas perturbados (DURIGAN e NOGUEIRA, 1990; GANDOLFI et al., 1995; MARCHIORI, 1997; LORENZI, 1998, 2002; CARVALHO, 2003; SANTOS, 2007).

Das 14 espécies encontradas, principalmente as seis discutidas acima, parecem estar bem estabelecidas, aparentemente exercendo suas funções vitais e reprodutivas dentro do normal (muitas apresentam flores e frutos). Destas seis, quatro constam no levantamento realizado pela EMBRAPA em áreas de pastagem ao longo de oito municípios do Estado do Rio de Janeiro (SOUTO et al, 2002).

A ordenação final das UAs (indivíduos), com base em suas características ambientais, apresentou um stress de 0,09 e captou $96,6 \%$ de proporção da variância expressa pelos dados. Além de a variância captada ter sido elevada, o stress final indica uma excelente representação da realidade, com poucas chances de interpretações errôneas (MCCUNE e GRACE, 2002). A sobreposição das elipses bivariadas de Gauss, sobre a ordenação final das UAs (Figura 2), representando os tratamentos (espécies), explicita a existência de uma diferenciação entre estes, a qual é confirmada pela MANOVA (Pillai trace $=0,281 ; \mathrm{F}=2,332 ; \mathrm{gl}=16,228 ; \mathrm{p}<$ $0,01)$. Este resultado confirma que há diferenciação quanto à ocorrência das espécies rústicas no ecossistema perturbado, e que esta parece ser consequência, ainda que parcialmente, dos fatores ecológicos aqui considerados. Destes, segundo a correlação de Pearson, aparentemente elevação, declividade e relevo são mais importantes para determinar a ocorrência das espécies rústicas na paisagem degradada (Tabela 4).

Em uma Floresta Estacional no município de Santa Maria - RS, a distribuição de grande parte das espécies estudadas, possui relação com a disposição das curvas de nível, quase sempre apresentando padrões diferenciados que acompanham a variação da topografia (GIEHL et al. 2007). Recentemente, tem crescido o número de trabalhos que evidenciam a importância da variação ambiental ao longo de gradientes, em particular referente ao relevo, para determinar a distribuição de espécies vegetais na paisagem (TUOMISTO et al. 2003; SVENNING et al., 2004, 2006; KARST et al. 2005; JONES et al., 2006, 2008; ZUQUIM et al. 2009; NETTESHEIM et al. 2010). Já é amplamente aceito hoje, por exemplo, o fato de que a elevação do terreno é um fator condicionante de diferentes padrões da vegetação. Entretanto, a altitude é um fator complexo de variação ambiental, uma vez que com ela podem variar a distribuição do solo, do clima, da precipitação, etc., devendo ser estas variáveis, quando possível, também independentemente consideradas (MONTEIRO e FISCH, 2005; BISPO et al., 2009). Outro importante fator de variação que está intimamente relacionada ao relevo, é a declividade do terreno. A declividade produz uma variedade de situações ambientais, como gradientes de umidade no solo entre o topo e base da vertente, controle do equilíbrio entre infiltração de água no solo e escoamento superficial, favorecimento do transporte de partículas de solo ao longo do perfil (controla a intensidade dos fluxos de matéria) e a variação 


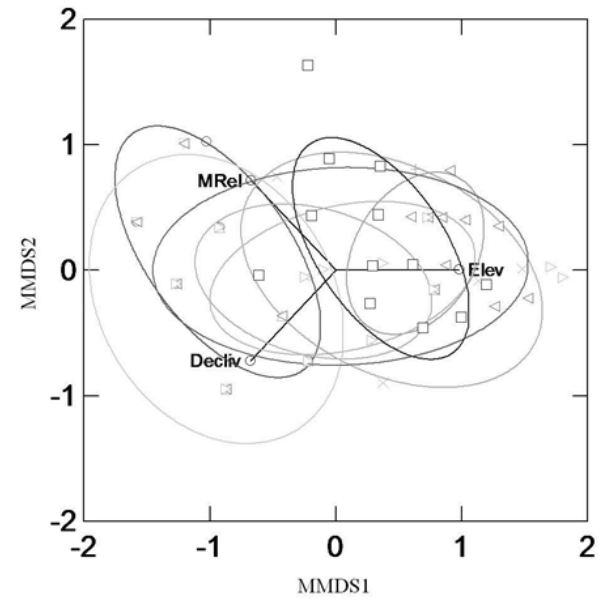

FIGURA 2: Configuração final dos indivíduos de cada espécie (unidades amostrais) pela MMDS (Análise Monotônica de Escalonamento Multidimensional). As elipses bivariadas de Gauss representam as espécies (tratamentos: Ap-Acacia polyphylla DC.; Cp- Cecropia pachystachya Trec.; Gg- Guarea guidonia (L.) Sleumer; Mh- Machaerium hirtum (Vell.) Stellfeld; Pd- Peltophorum dubium (Spreng.) Taub.; S1- Sparattosperma leucanthum (Vell.) Schum.; Tc- Tabebuia chrysotricha (Mart. ex DC.) Stan.; TlTabernaemontana laeta Mart.). Fatores ecológicos (Decliv - declividade; Elev Elevação; MRel - Microrrelevo) aparecem na forma de vetores que representam sua correlação de Pearson (maior do que 0,6) com cada um dos eixos.

FIGURE 2: Final configuration of individuals of each species (sampling units) for the MMDS (Monotonic Multi-Dimensional Scaling). The Gaussian bivariate ellipses represent the species (treatment: Ap- Acacia polyphylla DC.; Cp- Cecropia pachystachya Trec.; Gg- Guarea guidonia (L.) Sleumer; MhMachaerium hirtum (Vell.) Stellfeld; PdPeltophorum dubium (Spreng.) Taub.; Sl- Sparattosperma leucanthum (Vell.) Schum.; Tc- Tabebuia chrysotricha (Mart. ex DC.) Stan.; T1- Tabernaemontana laeta Mart.). Ecological factors (slope - slope; Elev - Elevation; MRel - Micro-Relief) appear in the form of vectors that represent their correlation (greater than 0.6) with each axis.

no tempo de incidência de radiação durante o ano (GANDOLFI, 2000; SCHMIDT et al., 2003; BISPO et al., 2009). Ainda assim, esse conhecimento vem sendo gerado, principalmente, em áreas que apre-
TABELA 4: Valores da correlação de Pearson entre a configuração final dos eixos da MMDS e os fatores ecológicos.

TABLE 4: Values of Pearson correlation between the final configuration of the axes of MMDS and ecological factors.

\begin{tabular}{lcccc}
\hline & Elevação & Declividade & Relevo & $\begin{array}{c}\text { Aff. } \\
\text { rochoso }\end{array}$ \\
\hline MMDS 1 & 0.98 & -0.68 & -0.67 & 0.14 \\
MMDS 2 & 0.005 & 0.72 & 0.71 & 0.03 \\
\hline
\end{tabular}

Em que: MMDS = Análise Monotônica de Escalonamento Multidimensional.

sentam melhor estado de conservação (TUOMISTO et al. 2003; SVENNING et al., 2004, 2006; BISPO et al., 2009; ZUQUIM et al. 2009; NETTESHEIM et al. 2010). Porém, este estudo demonstra que tais fatores podem ser relevantes para influenciar e determinar a ocorrência de espécies arbóreas rústicas também em áreas degradadas.

Analisando a Figura 2, a ocorrência de Tabebuia chrysotricha, Cecropia pachystachya, Guarea guidonia, Acacia polyphylla e Peltophorum dubium aparenta ser condicionada por pelo menos um dos fatores ecológicos considerados. Tabebuia chrysotricha aparenta ter uma tendência a ser condicionada pela elevação do terreno (Figura 2) e apresenta cerca de $53 \%$ dos indivíduos concentrados na classe 2 de elevação, entre 60-80m (Tabela 5); Cecropia pachystachya parece ser condicionada pela elevação e pelo microrrelevo (Figura 2), tendo cerca de $77,7 \%$ dos seus indivíduos concentrados nas classes 4 e $5(100-140 \mathrm{~m})$ e $56 \%$ nas calhas do relevo (Tabela 5); Guarea guidonia, apesar de também influenciada pela elevação, aparenta ter sofrido maior influência das condições de microrrelevo (Figura 2), pois 62,5\% dos indivíduos desta espécie concentraram-se em áreas de colúvio (Tabela 5); Acacia polyphylla também parece ser influenciada principalmente pelo microrrelevo (Figura 2) e 67\% dos indivíduos ocorrem em colúvios (Tabela 5); finalmente, Peltophorum dubium é a única espécie que parece ser mais condicionada pela declividade (Figura 2), onde 89\% dos indivíduos estão presentes nas áreas de maior declividade (Tabela 5) encontradas neste estudo (20 - 45\%). Tabebuia chrysotricha, por exemplo, possui sementes aladas e muito leves, passíveis de serem conduzidas pelo vento para as áreas mais baixas do relevo, onde tem maior ocorrência. No caso de Cecropia pachystachya, é possível que sua ocorrência em locais mais elevados seja consequência de sua síndrome de dispersão zoocó- 
rica, dado que os fragmentos que servem de fonte de propágulos e abrigo para a fauna nesta região estão em partes mais altas do relevo (SATO et al., 2008). Ainda outro exemplo seria Guarea guidonia, espécie tipicamente heliófita/seletiva higrófila muito associada às áreas úmidas (LORENZI, 2002), cuja ocorrência na área estudada parece ser mais condicionada pelo microrrelevo colúvio, que apresenta capacidade de acúmulo de umidade. Assim, é possível admitir que a distinta distribuição dessas espécies na paisagem seja consequência de cada uma possuir preferências e características particulares no que diz respeito à elevação, declividade $\mathrm{e}$ microrrelevo.

De acordo com o contexto do presente estudo, pode-se deduzir que, mesmo em espécies rústicas, ocorra variação no grau de especialização quanto a sua distribuição, podendo isso restringir sua abundância e importância na paisagem. Essa hipótese poderia explicar diferenças na representatividade de cada espécie na estrutura vegetal de ecossistemas perturbado. Porém, existem outros fatores que, não necessariamente os do meio físico, sejam determinantes na ocorrência e distribuição espacial destas espécies no ecossistema analisado. A estrutura e biodiversidade das comunidades são reguladas por fatores abióticos como clima e geomorfologia (MELO e LIMA, 2007), e fatores bióticos como composição florística e estágio sucessional de espécies que compõem determinado ecossistema (PEREIRA et al., 2004).

Tabernaemontana laeta, Sparattosperma leucanthum e Machaerium hirtum foram as três espécies mais importantes na atual estrutura do ecossistema estudado e não demonstraram tendência por nenhuma preferência ambiental relacionada aos fatores ecológicos analisados (Figura 2).

No caso dessas espécies, é possível que isso denote um caráter mais generalista quanto à variação da elevação, declividade e microrrelevo em ecossistemas perturbados, facilitando seu estabelecimento e desenvolvimento em relação às demais espécies aqui apresentadas. Ainda assim, devido à elevada variabilidade do relevo e, consequentemente, de fatores ecológicos potencialmente condicionantes para espécies rústicas em áreas degradadas,

TABELA 5: Ocorrência dos indivíduos de cada espécie de acordo com as diferentes classes de cada um dos três principais fatores ecológicos incluídos na análise (dados referentes a afloramentos rochosos não aparecem devido a sua baixa relevância na análise).

TABLE 5: Occurrence of individuals of each species according to the different classes of each of the three main ecological factors included in the analysis (the data for rock outcrops do not appear due to their low relevance in the analysis).

\begin{tabular}{|c|c|c|c|c|c|c|c|c|c|c|c|c|c|c|}
\hline & \multicolumn{8}{|c|}{ Classe de elevação } & \multicolumn{3}{|c|}{$\begin{array}{l}\text { Classe de } \\
\text { declividade }\end{array}$} & \multicolumn{3}{|c|}{ Microrrelevo } \\
\hline & 1 & 2 & 3 & 4 & 5 & 6 & 7 & 8 & A & $\mathrm{B}$ & $\mathrm{C}$ & Calha & Colúvio & Rampa \\
\hline Tabernaemontana laeta Mart. & 0 & 7 & 5 & 6 & 6 & 4 & 2 & 0 & 0 & 14 & 16 & 12 & 17 & 1 \\
\hline $\begin{array}{l}\text { Sparattosperma leucanthum (Vell.) } \\
\text { Schum. }\end{array}$ & 0 & 7 & 5 & 7 & 3 & 4 & 1 & 1 & 0 & 14 & 14 & 13 & 7 & 8 \\
\hline Machaerium hirtum (Vell.) Stellfeld. & 0 & 10 & 3 & 2 & 2 & 0 & 0 & 0 & 0 & 11 & 6 & 9 & 8 & 0 \\
\hline $\begin{array}{l}\text { Tabebuia chrysotricha (Mart. ex DC.) } \\
\text { Stan. }\end{array}$ & 0 & 8 & 2 & 3 & 1 & 1 & 0 & 0 & 1 & 6 & 8 & 6 & 5 & 4 \\
\hline Cecropia pachystachya Trec. & 0 & 2 & 0 & 4 & 3 & 0 & 0 & 0 & 0 & 4 & 5 & 5 & 2 & 2 \\
\hline Peltophorium dubium (Spreng.) Taub. & 0 & 2 & 2 & 2 & 2 & 1 & 0 & 0 & 0 & 1 & 8 & 4 & 2 & 3 \\
\hline Guarea guidonia (L.) Sleumer & 0 & 1 & 1 & 1 & 2 & 1 & 1 & 1 & 0 & 4 & 4 & 2 & 5 & 1 \\
\hline Acacia polyphylla DC. & 0 & 3 & 1 & 0 & 0 & 1 & 1 & 0 & 0 & 2 & 4 & 1 & 4 & 1 \\
\hline Psidium guajava $\mathrm{L}$. & 0 & 1 & 0 & 0 & 1 & 1 & 0 & 0 & 0 & 1 & 2 & 0 & 0 & 3 \\
\hline Coutarea hexandra (Jacq.) K. Schum. & 0 & 0 & 0 & 2 & 0 & 0 & 0 & 0 & 0 & 2 & 0 & 2 & 0 & 0 \\
\hline Apuleia Leiocarpa (Vogel) J.F. Macbr. & 0 & 0 & 0 & 0 & 0 & 0 & 1 & 0 & 0 & 0 & 1 & 0 & 1 & 0 \\
\hline Cupania oblongifolia Mart. & 0 & 0 & 0 & 1 & 0 & 0 & 0 & 0 & 0 & 0 & 1 & 1 & 0 & 0 \\
\hline Myrocarpus fastigiatus Allemao & 0 & 0 & 0 & 0 & 1 & 0 & 0 & 0 & 0 & 0 & 1 & 0 & 1 & 0 \\
\hline Sapium glandulatum (Vell.) Pax & 0 & 0 & 0 & 0 & 0 & 0 & 1 & 0 & 0 & 0 & 1 & 0 & 1 & 0 \\
\hline Total & 0 & 41 & 19 & 28 & 21 & 13 & 7 & 2 & 1 & 59 & 71 & 55 & 53 & 23 \\
\hline
\end{tabular}

Em que: Classes de declividade observadas: $\mathrm{A}=$ suavemente ondulado; $\mathrm{B}=$ ondulado; $\mathrm{C}=$ fortemente ondulado. 
gerando diferentes configurações estruturais, é importante que este tipo de estudo seja reproduzido em outros locais, considerando o maior número de variáveis ambientais possível.

\section{CONCLUSÕES}

Foram levantadas e identificadas 14 espécies arbóreas rústicas que conseguiram se estabelecer espontaneamente em uma vertente considerada perturbada por usos pretéritos na bacia do rio Cacaria. A análise de parte dessas espécies permitiu identificar a existência de alguns padrões de distribuição ao longo da área degradada em função dos fatores ecológicos de elevação, declividade e microrrelevo. É possível que esses padrões ajudem a elucidar algumas das questões referentes à variação estrutural de espécies rústicas em ecossistemas perturbados. Entretanto, dada a elevada variação de fatores ecológicos potencialmente condicionantes nestes ambientes, é necessário reproduzir estudos como este em outras áreas igualmente impactadas por ações antrópicas. Informações como as geradas por este estudo são importantes bases para se gerar futuras diretrizes na restauração de ecossistemas degradados. Conhecer mais sobre os fatores que condicionam a ocorrência de espécies rústicas em áreas perturbadas pode ajudar a usar estas espécies como importantes facilitadoras no processo de recuperação. Não só estas plantas apresentam maior facilidade de se estabelecer, como ocorrem, geralmente, isoladas na área perturbada e por isso servem de locais estratégicos para pouso entre fragmentos, contribuindo para a entrada de novas espécies vegetais. Assim, poder utilizar o conhecimento sobre as preferências ecológicas de espécies arbóreas rústicas para favorecer o seu estabelecimento em ecossistemas perturbados parece ser uma boa opção como ponto de partida para a facilitação do desenvolvimento da regeneração natural.

\section{AGRADECIMENTOS}

Aos Professores Márcio Rocha Francelino, André Felippe Nunes-Freitas (IF/UFRRJ), Marilena de Menezes Silva Conde (IB/UFRRJ); à CAPES pela Bolsa de Estudos; ao Sítio Monumento e EPTCA Medical Devices pelo apoio logístico.

\section{REFERÊNCIAS BIBLIOGRÁFICAS}

APG (Angiosperm Phylogeny Group) II. An update of the Angiosperm Phylogeny Group classification for the orders and families of flowering plants: APG II. Botanical Journal of the Linneam Society. London, v. 141, p. 399 - 436, 2003.

BISPO, P. C.; VALERIANO, M. M.; KUPLICH, T. M. Variáveis geomorfométricas locais e sua relação com a vegetação da região do interflúvio MadeiraPurus (AM-RO). Acta Amazônica, Manaus, v. 39, n. 1, mar. 2009.

CARVALHO, P. E. R. Espécies Arbóreas Brasileiras. Colombo: EMBRAPA Florestas, 2003. v. $1.1039 \mathrm{p}$.

CORTINES, E.; VALCARCEL, R. Influence of pioneer-species combinations on restoration of disturbed ecosystems in the Atlantic Forest, Rio de Janeiro, Brazil. Revista Árvore. v. 33, n. 5, p. 925934, jul./ago., 2009.

DEAN, W. A ferro e fogo: a história e a devastação da Mata Atlântica brasileira. São Paulo: Companhia das Letras, $1996.491 \mathrm{p}$.

DURIGAN, G.; NOGUEIRA, J.C.B. Recomposição de Matas Ciliares. São Paulo: Instituto Florestal, 1990. 14 p. (IF - Série registros, v. 4).

EMBRAPA - EMPRESA BRASILEIRA DE PESQUISA AGROPECUÁRIA. Análises físicas. In: Manual de métodos de análise de solo. 2. ed. Rio de Janeiro: Centro Nacional de Pesquisas de Solo, 1997. P. 1997. P. 1-79. (Embrapa - CNPS: Documentos, 1).

ESPINOLA, L. A.; JULIO JUNIOR, H. F. Espécies invasoras: conceptos, modelos y atributos. INCI, v. 32, n. 9, p.580-585. Sept. 2007.

GANDOLFI, S. História natural de uma Floresta Estacional Semidecidual no município de Campinas (São Paulo, Brasil). 2000. Tese (Doutorado) - Universidade Estadual de Campinas, Campinas, 2000.

GANDOLFI, S.; LEITÃO FILHO, H. F.; BEZERRA, C. L. F. Estudo florístico e caráter sucessional das espécies arbustivo-arbóreas de uma floresta mesófila semidecidual no município de Guarulhos, SP. Revista Brasileira de Biologia, v. 55, n. 4, p. 753-767. 1995.

GENTRY, A. H. Bignoniaceae - Part II (Tribe Tecomeae). Flora Neotropica. Monograph, v. 25, p. 1-370. 1992.

GIEHL, E. L. H.; BUDKE, J. C.; ATHAYDE, E. A. Distribuição espacial de espécies arbóreas em uma floresta estacional em Santa Maria, Sul do Brasil. Pesquisas Botânica, São Leopoldo, n. 58, p. 215226. 2007.

IBGE. Vocabulário básico de recursos naturais e 
meio ambiente. Rio de Janeiro: IBGE, 2004.

JONES, M. M. et al. Effects of mesoscale environmental heterogeneity and dispersal limitation on floristic variation in rain forest ferns. Journal of Ecology, v. 94, p. 181-195. 2006.

JONES, M. M. et al. Explaining variation in tropical plant community composition: influence of environmental and spatial data quality. Oecologia. v. 155 , p. 593-604. 2008.

KARST, J.; GILBERT, B.; LECHOWICZ, M. J. Fern community assembly: The roles of chance and the environment at local and intermediate scale. Ecology. v. 86, p. 2473-2486. 2005

LONGHI, S.J. etal. Fatores ecológicos determinantes na ocorrência de Araucaria angustifolia e Podocarpus lambertii, na Floresta Ombrófila Mista da FLONA de São Francisco de Paula, RS, Brasil. Ciência Rural, Santa Maria, Online. http://www. scielo.br/pdf/cr/v40n1/a399cr1265.pdf . Acessado em 26 fev. 2010.

LORENZI, H. Árvores Brasileiras: manual de identificação e cultivo de plantas arbóreas nativas do Brasil. Nova Odessa: Plantarum, 1998. v. 1-2.

LORENZI, H. Árvores Brasileiras: manual de identificação e cultivo de plantas arbóreas nativas do Brasil. Nova Odessa: Plantarum, 2002. v. 2-2.

MARCHIORI, J. N. C. Dendrologia das angiospermas: Leguminosas. Santa Maria. Ed. da UFSM, 1997.

MATOZINHOS, C. N. e KONNO, T. U. P. Apocynaceae S.L. na Reserva Biológica da Represa do Grama, Descoberto, MG,Brasil. Rodriguésia, v. 59, n. 1, p. 087-098. 2008.

MATTEUCCI,S.D.; COLMA,A.Metodologiapara el estudio de la vegetación. Washington, Secretaria General de la OEA, 1982. 169 p.

MCCUNE, B.; GRACE, J. B. Analysis of Ecological Communities. MJM, Software Design. Gleneden Beach, Oregon, 2002.

MELO, C. E.; LIMA, J. D. Diversidade de espécies e influência de fatores estocásticos na regulação da ictiofauna em lagos de meandros na bacia do Rio das Mortes - Mato Grosso, Brasil. Brazilian Journal of Ecology, v. 1-2, p. 20-25, 2007.

MONTEIRO, E. A.; FISCH, S. T. V. Estrutura e padrão espacial das populações de Bactris setosa Mart. e B. hatschbachii Noblick ex A. Hend (Arecaceae) em um gradiente altitudinal, Ubatuba (SP). Biota Neotropica, v. 5, n. 2. 2005.

MORI, S. A. et al. Manual de manejo do herbário fanerogâmico. Itabuna, CEPLAC, 1985. 97 p.

NETTESHEIM, F. C. et al. Influence of environmental variation on Atlantic Forest treeshrub-layer phytogeography in southeast Brazil. Acta Botânica Brasílica, v. 24, n. 2. 2010.

PEREIRA, M. C. A.; CORDEIRO, S. Z.; ARAUJO,D. S. D. Estrutura do estrato herbáceo na formação aberta de Clusia do Parque Nacional da Restinga de Jurubatiba, RJ, Brasil. Acta bot. bras. v. 18, n. 3, p. 677-687. 2004.

PEREIRA, P. H. e MANSANO, V. F. Estudos taxonômicos da tribo Tecomae (Bignoniaceae) no Parque Nacional do Itatiaia, Brasil. Rodriguésia, $\mathrm{v}$. 59, n. 2, p. 265-289. 2008.

POTT, A.; POTT, V. J. Plantas nativas para recuperação de áreas degradadas e reposição da vegetação no Mato Grosso do Sul.. Campo Grande: EMBRAPA gado de corte, 2002. (Comunicado Técnico - EMBRAPA. n. 75).

RODRIGUES, L. A. et al. Efeitos de solos e topografia sobre a distribuição de espécies arbóreas emum fragmento de floresta estacional semidecidual, em Luminárias, MG. Revista Árvore, Viçosa, v. 31 , n. 1, p. 25-35, 2007.

SANTOS, F.S. Biometria, germinação e qualidade fisiológica de sementes de Tabebuia chrysotricha (Mart. Ex A. Dc.) Standl. provenientes de diferentes matrizes. 2007. Dissertação (mestrado) - Universidade Estadual Paulista, Faculdade de Ciências Agrárias e Veterinárias. Jaboticabal, 2007. SATO, T. M.; CAMARGO, F.; NOGUEIRA, A. C. Frugivoria de morcegos (Mammalia, Chiroptera) em Cecropia pachystachya (Urticaceae) e seus efeitos na germinação das sementes. Papeis Avulsos de Zoologia - Museu de Zoologia da Universidade de São Paulo. v. 48, n. 3, p. 19-26, 2008.

SCHMIDT, J.; EVANS, I. S.; BRINKMANN, J. Comparison of polynomial models for land surface curvature calculation. International Journal of Geographical Information Science, v. 17, n. 8, p. 797-814. 2003.

SOUTO, S. M. et al. Levantamento de árvores em pastagens no municípios das regiões Serrana, Litorânea, Centro-Sul e Sul do Estado do Rio de Janeiro. Seropédica: EMBRAPA Agrobiologia, 2002. (EMBRAPA Agrobiologia, Documentos, $n$. 152 ).

SVENNING, J. C. et al. Ecological determinism in plant community structure across a tropical forest landscape. Ecology, v. 85, p. 2526 - 2538. 2004.

SVENNING, J. C. et al. The relative roles of environment, history and local dispersal in controlling the distributions of common tree and shrub species in a tropical forest landscape, Panama. 
Journal of Tropical Ecology, v. 22, p. 575-586. variation of Western Amazonian forests. Science, v. 2006. 299, p.241-244. 2003.

SYSTAT for Windows. Version 11 [S.I.]: Systat ZUQUIM, G. et al. Distribution of pteridophyte Software, 2005. communities along environmental gradients in TUOMISTO, H.; RUOKOLAINEN, K.; YLI- Central Amazonia, Brazil. Biodiversity and HALLA, M. Dispersal, environmental, and floristic Conservation, v. 18, p. 151-166. 2009. 